\title{
Synthesis and Mesophase Characterization of Liquid Crystalline Polyesters With Bulky, Rigid, Lateral Substituents
}

\author{
N. KHAN, D. M. PRICE, and Z. BASHIR* \\ Courtaulds Research, 72 Lockhurst Lane, Coventry CV6 5RS, United Kingdom
}

\begin{abstract}
SYNOPSIS
The synthesis and characterization of aromatic polyesters containing various symmetrically di-substituted hydroquinone monomers is described. The homopolyesters made from these monomers and terephthalic acid (TA) did not melt. Copolyesters of TA with mono- and di-substituted hydroquinone formed liquid crystalline melts. Optical microscopy showed schlieren, marbled, and droplet textures characteristic of the nematic phase. DSC experiments were also in accord with mesophase formation as multiple transitions characteristic of first-order phase changes were found. In general, the crystal-nematic transition was about $300^{\circ} \mathrm{C}$, whereas the nematic-isotropic change was over $400^{\circ} \mathrm{C}$. All the polyesters were prone to decomposition near or above the isotropization temperature. (C) $1994 \mathrm{John}$ Wiley \& Sons, Inc.

Keywords: thermotropic $\bullet$ liquid crystalline $\bullet$ polyesters $\bullet$ frustrated chain packing $\bullet$ nematic - optical textures $\bullet$ DSC transitions
\end{abstract}

\section{INTRODUCTION}

It is well known that certain aromatic polyesters can show thermotropic liquid crystalline behavior. The great interest in these materials stems from the facility with which they can be oriented and spun into high modulus fibers or injection molded into articles that have superior mechanical properties when compared with conventional thermoplastics.

One of the prerequisites of forming liquid crystalline phases is that the molecules be rod-like (or less commonly disk-like) in shape. Polyesters based on $100 \%$ p-hydroxy benzoic acid (p-HBA) or terephthalic acid (TA) and hydroquinone (HQ) (polymers I and II, Table I) are infusible and insoluble. This is because a very rigid chain leads to a low entropy of fusion, which raises the melting point above the degradation temperature.

Liquid crystal polymers (LCPs) with very high glass transition and melting temperatures are now commercially available and have applications that make them desirable. For instance Xydar (manufactured by Dartco) is a copolyester based on 4,4'-

* To whom correspondence should be addressed.

Journal of Polymer Science: Part B: Polymer Physics, Vol. 32, 2509-2518 (1994) (C) 1994 John Wiley \& Sons, Inc. biphenol, TA, and p-HBA; polymers such as this, however, have a crystal-nematic transition over $400^{\circ} \mathrm{C}^{1}$ and this can limit their use in conventional processing machinery. Thus, there is also a drive to make thermotropic LCPs with lower processing temperatures. There have been a number of investigations aimed at lowering the crystal melting point to temperatures of $300^{\circ} \mathrm{C}$ or below, while maintaining the liquid crystalline behavior.

There are several synthetic strategies to reduce the melting temperatures of rigid-rod polymers and yet form liquid crystal phases. One option is to introduce flexible alkyl spacers between the aromatic units. ${ }^{2}$ Another method is to include kinks such as meta linkages, which disrupt the chain linearity of purely para-aromatic sequences. ${ }^{2}$ Appending flexible side chains to create "hairy-rod" thermotropic polymers is another avenue explored by Ballauf and Schmidt. ${ }^{3}$ Griffin and Cox $^{2}$ have suggested that including aromatic units with bulky substituents on the chain backbone frustrates chain packing and this lowers the crystallinity and depresses the melting point below the degradation temperature. In this work, the concept of frustrated chain packing will be used to synthesize polyesters that exhibit liquid crystalline behavior at relatively moderate processing temperatures. 
Table I

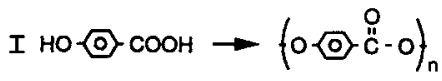

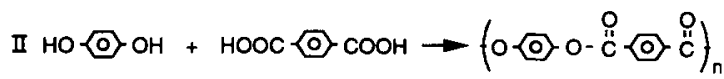

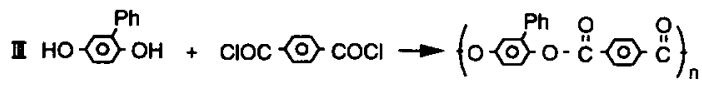

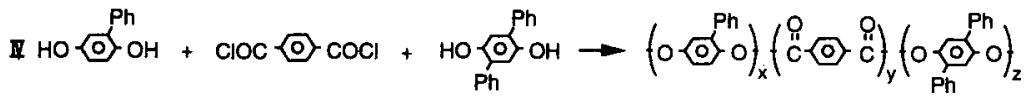

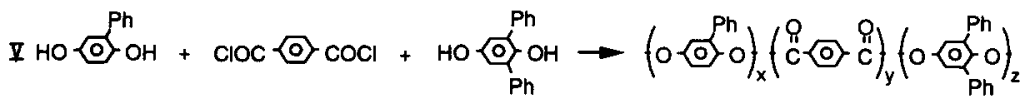

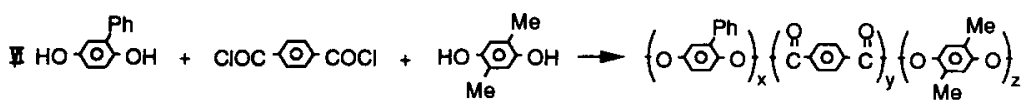

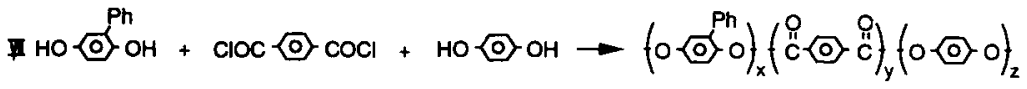

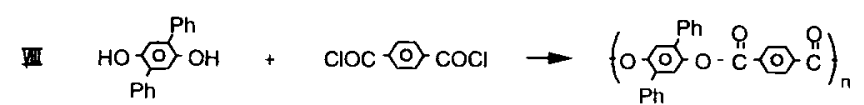

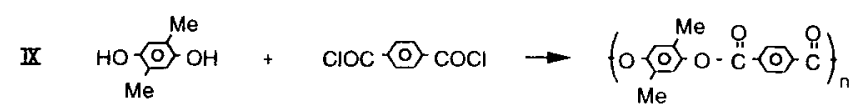

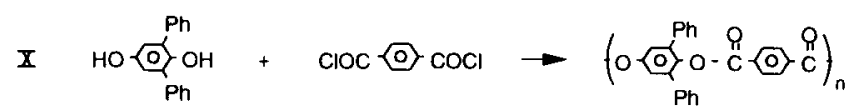

The literature contains examples of aromatic polyesters with methyl-, bromo-, chloro-, and methoxy-substituted hydroquinone. ${ }^{4}$ Substitution of the hydroquinone with a phenyl group to form phenyl p-phenylene terephthalate (polyester III in Table I) was disclosed by Payet. ${ }^{5}$ Jackson has indicated the superior thermal stability of the phenyl-substituent compared with the chloro or methyl groups in such polyesters. ${ }^{6}$ It was reported that a liquid crystal melt was formed, and fibers based on this had excellent mechanical properties. ${ }^{5,7}$ According to Krigbaum and Tanaka, the phenyl-substituted polyester III has a $\mathrm{T}_{\mathrm{g}}$ of $140^{\circ} \mathrm{C}$, a crystal-nematic transition of $350^{\circ} \mathrm{C}$, and a clearing temperature of $455-460^{\circ} \mathrm{C} ;^{8}$ however, there is some variability in the transition temperatures cited by different authors for the same polymer. The analogue of this polymer, namely the polyester with the phenyl substituent on the terephthalic acid instead of the hydroquinone, has also been disclosed by Harris, ${ }^{9}$ and is reported by different authors to have a crystalnematic transition of $300^{\circ} \mathrm{C}^{9}$ or $265^{\circ} \mathrm{C} .^{10}$

In this work, we have examined the synthesis and phase behavior of homopolyesters of terephthalic acid and some new symmetrically di-substituted hydroquinones (polymers VIII to $\mathrm{X}$, Table I), as well as copolyesters of terephthalic acid, phenyl hydroquinone, and the new di-substituted hydroquinones (polymers IV-VI, Table I).

\section{EXPERIMENTAL}

\section{Synthesis of Monomers}

Several of the monomers and intermediates were commercially available. Terephthaloyl chloride, hydroquinone ( 1,4 dihydroxy benzene), phenyl hydroquinone, 2,6-diphenylphenol, and 2,5-dimethylphenol were purchased from Aldrich. The 2,5-diphenylbenzoquinone was purchased from Lancaster Synthesis.

However, the di-substituted hydroquinones had to be synthesized. The reaction schemes are shown in Table II and the preparation details of the monomers and intermediates needed to make polyesters IV-VI and VIII-X are given below.

\section{2,5-Diphenylhydroquinone (2,5-DPHQ)}

Scheme A in Table II shows the reaction involved. The 2,5-diphenyl-1,4-benzoquinone ( $5 \mathrm{~g}, 0.019 \mathrm{~mol}$ ) was dissolved in boiling ethanol $(50 \mathrm{ml})$, and a five- 
fold excess of sodium hydrosulphite $(16.73 \mathrm{~g}, 0.096$ mol, $10 \%$ aqueous solution ) was added with vigorous stirring. The product precipitated immediately. The mixture was stirred for $0.5 \mathrm{~h}$ and then the product was filtered, washed with water several times, and dried in vacuum $\left(22^{\circ} \mathrm{C} / 750\right.$ mbar $)$. The product was recrystallized from toluene/petroleum ether (bp 40: $60^{\circ} \mathrm{C}$ ) to give $4.49 \mathrm{~g}$ ( $88 \%$ ) of the purified compound with $\mathrm{mp} 212-214^{\circ} \mathrm{C}$, ir $\nu_{\max } 1480,1408,1210,1148$, $1144,816,761,698 \mathrm{~cm}^{-1}, \mathrm{NMR} \delta \mathrm{CDCl}_{3}(80 \mathrm{MHz})$ 6.84 ( $2 \mathrm{H}, \mathrm{s}, \mathrm{Ar}$ ), 7.19-7.65 (10 H, m, Ar), and 8.35 $(\mathrm{s}, \mathrm{OH})$.

\section{2,6-Diphenylbenzoquinone}

Step 1 of scheme B in Table II shows the reaction involved in forming this intermediate. Jones reagent [prepared from sodium dichromate ( $80 \mathrm{~g})$, concentrated sulphuric acid $(50 \mathrm{ml})$, and water $(120 \mathrm{ml})]$ was added dropwise at $0^{\circ} \mathrm{C}$ to a stirred solution of 2,6-diphenylphenol ( $15 \mathrm{~g}, 0.057 \mathrm{~mol}$ ) in ether ( 200 $\mathrm{ml}$ ), and then the reaction mixture was stirred at room temperature for $7 \mathrm{~h}$. The reaction mixture was extracted with ether $(4 \times 100 \mathrm{ml})$ and the combined extracts were washed with $20 \%$ aqueous sodium hydrogen carbonate $(2 \times 50 \mathrm{ml})$, followed by water, and then dried over $\mathrm{MgSO}_{4}$. The solvent was evaporated to give an orange solid that was recrystallized from methanol to give $8.65 \mathrm{~g}(62 \%)$ of pure product, with $\mathrm{mp} 135-145^{\circ} \mathrm{C}$, ir $\nu_{\max } 1660$ (CO) and 1647 (CO) $\mathrm{cm}^{-1}, \mathrm{NMR} \delta \mathrm{CDCl}_{3}(80 \mathrm{MHz}) 6.91(2 \mathrm{H}, \mathrm{s})$, and $7.47(2 \mathrm{H}, \mathrm{m}, \mathrm{Ar})$.

\section{2,6-Diphenylhydroquinone (2,6 DPHQ)}

Step 2 of scheme B in Table II shows the reaction involved. The 2,6-diphenyl-1,4-benzoquinone $(5.0 \mathrm{~g}$, $0.019 \mathrm{~mol}$ ) produced in the previous reaction was dissolved in boiling ethanol $(50 \mathrm{ml})$, and a fivefold excess of sodium hydrosulphite $(16.73 \mathrm{~g}, 0.09 \mathrm{~mol}$, $10 \%$ aqueous solution) was added with vigorous stirring. The product precipitated immediately. The mixture was stirred for $0.5 \mathrm{~h}$ and then the product was filtered, washed with water several times, and dried in vacuum. The product was recrystallized from toluene to give $4.12 \mathrm{~g}(81 \%)$ of pure product, with $\mathrm{mp} 185-187^{\circ} \mathrm{C}$, ir $\nu_{\max } 3482(\mathrm{OH}) \mathrm{cm}^{-1}$, NMR $\mathrm{CDCl}_{3}(80 \mathrm{MHz}) 5.57(\mathrm{OH}, \mathrm{s}), 6.89(2 \mathrm{H}, \mathrm{s}, \mathrm{Ar})$, 7.39-7.64 (10 H, m, Ar ), and 8.4 (OH, s).

\section{2,5-Dimethylbenzoquinone}

Step 1 of scheme $\mathrm{C}$ in Table II shows the reaction involved in the formation of this intermediate. Jones reagent [prepared from sodium dichromate (165 g), concentrated sulphuric acid $(110 \mathrm{ml})$, and water $(235 \mathrm{ml})$ ] was added dropwise at $0^{\circ} \mathrm{C}$ to a stirred solution of 2,5-dimethylphenol ( $30 \mathrm{~g}, 0.11 \mathrm{~mol})$ in ether $(500 \mathrm{ml})$, and then the reaction mixture was stirred at room temperature for $12 \mathrm{~h}$. The reaction mixture was extracted with ether $(4 \times 100 \mathrm{ml})$ and the combined extracts were washed with $20 \%$ aqueous sodium hydrogen carbonate $(2 \times 50 \mathrm{ml})$, followed by water, and then dried over $\mathrm{MgSO}_{4}$. The solvent was evaporated to give an orange solid that was recrystallized from methanol to give $15.71 \mathrm{~g}$ (47\%) of pure product $\mathrm{mp} 130-132^{\circ} \mathrm{C}$, ir $\nu_{\max } 1660$ (CO), and $1647(\mathrm{CO}) \mathrm{cm}^{-1}, \mathrm{NMR} \delta \mathrm{CDCl}_{3}(80 \mathrm{MHz})$ $2.0(6 \mathrm{H}, \mathrm{d}, \mathrm{Me})$, and $6.55(2 \mathrm{H}, \mathrm{m}, \mathrm{CH}=)$.

\section{2,5-Dimethylhydroquinone (2,5-DMHQ)}

Step 2 of scheme $\mathrm{C}$ in Table II shows the reaction involved. The 2,5-dimethylbenzoquinone (7.5 g, $0.028 \mathrm{~mol}$ ) from the previous reaction was dissolved in boiling ethanol $(150 \mathrm{ml})$, and a fivefold excess of

Table II. Reaction Schemes for Monomer Preparation

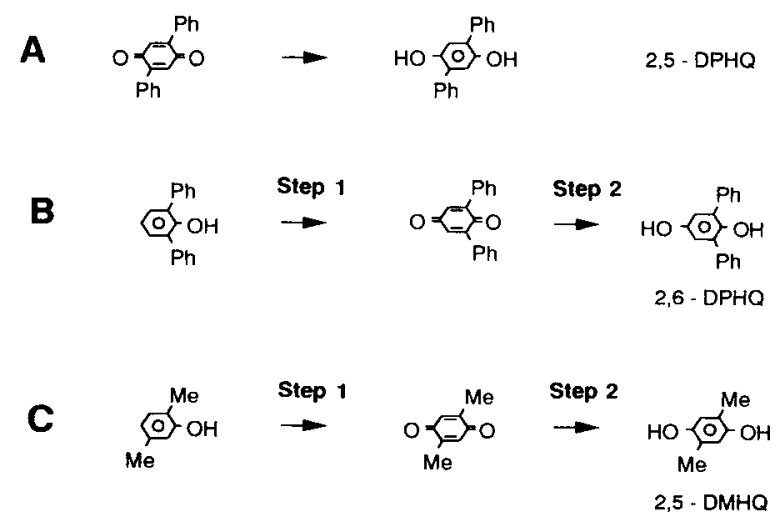


sodium hydrosulphite ( $48 \mathrm{~g}, 10 \%$ aqueous solution) was added with vigorous stirring. The mixture was stirred at room temperature for 3-4 h. Ethanol was removed under reduced pressure and the resulting aqueous solution was extracted several times with ether. The combined extracts were dried over $\mathrm{MgSO}_{4}$ and solvent was evaporated to give a white solid that was recrystallized from ether:petroleum ether (40: 60 ) to give $5.79 \mathrm{~g} \mathrm{(76 \% )} \mathrm{of} \mathrm{purified} \mathrm{product} \mathrm{with} \mathrm{mp}$ $225-228^{\circ} \mathrm{C}$, ir $\nu_{\max } 3232(\mathrm{OH}) \mathrm{cm}^{-1}, \mathrm{NMR} \delta \mathrm{CDCl}_{3}$ $(80 \mathrm{MHz}), 2.1(6 \mathrm{H}, \mathrm{s}, \mathrm{me}), 6.5(\mathrm{~s}, 2 \mathrm{H}, \mathrm{Ar})$, and $7.5(\mathrm{~s}, \mathrm{OH})$.

\section{Synthesis of Polymers}

The monomer compositions used to make the homoand copolyesters are shown in Table III. Note that instead of using terephthalic acid, terephthaloyl chloride was used due to its higher reactivity. However, in the discussion, these polyesters will be considered as being derived from the parent terephthalic acid.

The desired proportions of terephthaloyl chloride and derivatives of hydroquinone were placed into a polymerization flask equipped with a magnetic stirrer, a gas inlet/outlet, and a vacuum inlet. The polymerization flask was evacuated and then filled with nitrogen. This cycle was repeated four times. A slow stream of nitrogen was maintained in the flask to carry off the hydrogen chloride produced during the polymerization. The flask was heated in an oil bath to $190^{\circ} \mathrm{C}$. To compensate for the melt becoming more viscous, the temperature was raised slowly to $240^{\circ} \mathrm{C}$. When it was impossible to stir the melt further, vacuum ( $750 \mathrm{mbar}$ ) was applied and the melt was kept at this temperature for $5 \mathrm{~h}$. The flask was cooled to room temperature, the polymer was me- chanically crushed, extracted with acetone, and dried at $100^{\circ} \mathrm{C}$.

In one case ( polyester IV), two variants, a higher and a lower molecular weight material, were synthesized. Polyester IV A will denote the lower molecular weight and IV B will denote the higher molecular weight material. IV A was synthesized by reducing the standard polymerization time of $5 \mathrm{~h}$ to $2.5 \mathrm{~h}$ and conducting the entire reaction at $190^{\circ} \mathrm{C}$.

\section{Polarizing Optical Microscopy}

All samples were examined with a Zeiss Axioplan polarizing microscope, equipped with hot-stage lenses. A Linkam hot-stage and allied temperature controller were used to heat and cool the sample at a controlled rate.

The general procedure adopted to obtain the best textures was to heat the sample up to the clearing temperature with the fastest heating rate available $\left(99^{\circ} \mathrm{C} / \mathrm{min}\right)$ and then to cool the sample immediately. This minimized degradation and gave the best textures. In cases where the isotropization led to charring, the sample was heated close to the isotropization transition very rapidly, and then cooled to a lower temperature where the polymer was still fluid.

\section{Differential Scanning Calorimetry (DSC)}

A Mettler DSC 30 was used in conjunction with a TC11 controller. Data analysis was performed on an IBM microcomputer. The DSC cell was calibrated for temperature and enthalpy response according to the melting points and heats of fusion of pure indium, tin, zinc, and lead.

Table III. The Monomer Compositions of the Polyesters Used in This Work

\begin{tabular}{|c|c|c|c|c|c|c|}
\hline \multirow[b]{2}{*}{ Polymer } & \multicolumn{6}{|c|}{ Molar Percentage of } \\
\hline & TPCL & PHQ & 2,5-DPHQ & 2,6-DPHQ & 2,5-DMHQ & $\mathrm{HQ}$ \\
\hline III & 50 & 50 & - & - & - & - \\
\hline IV & 50 & 43.75 & 6.25 & - & - & - \\
\hline $\mathrm{V}$ & 50 & 43.75 & - & 6.25 & - & - \\
\hline VI & 50 & 43.75 & - & - & 6.25 & - \\
\hline VII & 50 & 37.50 & - & - & - & 12.5 \\
\hline VIII & 50 & - & 50 & - & - & - \\
\hline IX & 50 & - & - & - & 50 & - \\
\hline $\mathrm{x}$ & 50 & - & - & 50 & - & - \\
\hline
\end{tabular}

$\mathrm{TPCL}=$ terephthaloyl chloride; $\mathrm{PHQ}=$ phenyl hydroquinone; $2,5-\mathrm{DPHQ}=2,5$-diphenyl hydroquinone; 2,6-DPHQ $=2,6$-diphenyl hydroquinone; 2,5-DMHQ = 2,5-dimethyl hydroquinone; $\mathrm{HQ}=$ hydroquinone. 
In the first series of experiments, the heating thermograms of all the polyesters were recorded at the fastest practical heating rate possible $\left(40^{\circ} \mathrm{C} /\right.$ $\mathrm{min}$ ) to minimize degradation. From the optical microscopy, two transitions were expected, and hence the upper temperature limit was chosen accordingly for each polymer. After the second high temperature transition, the sample was cooled at $40^{\circ} \mathrm{C} / \mathrm{min}$ and the cooling thermogram was recorded.

In the second set of experiments, the most thermally stable polyester IV A (Table I) was selected for studies on the effect of heating and cooling rates. Thermograms were recorded between 20 to $400^{\circ} \mathrm{C}$ and 400 to $20^{\circ} \mathrm{C}$, at heating and cooling rates of $\pm 5^{\circ} \mathrm{C} / \mathrm{min}, \pm 10^{\circ} \mathrm{C} / \mathrm{min}$, and $\pm 40^{\circ} \mathrm{C} / \mathrm{min}$.

\section{RESULTS AND DISCUSSION}

Table I shows the polymers synthesized in this work (III-X). The homopolymers of terephthalic acid and the symmetrically di-substituted hydroquinones (VIII to X, Table I) did not melt and so no further work was carried out on these.

The remaining copolyesters III-VII melted and showed LC phases. The composition of the ingredients is shown in Table III. Due to the insolubility of the materials in common non-toxic solvents, molecular weight characterisation was not performed. However, all the materials had mechanical properties consistent with high molecular weight polymer. All were film and fiber forming. Dumbells were made from polymers III-VII by compression molding. In addition, polyester IV A was injection molded to produce a screw-driver handle.

Polymer III is discussed fairly extensively in the literature ${ }^{4-8}$ and hence it will not be considered in detail here, but it will be used as a reference for comparison of the other polyesters. It is quoted by one set of authors as having a crystal-nematic transition at $350^{\circ} \mathrm{C}$ and a clearing temperature of $460^{\circ} \mathrm{C} .^{8}$ Our DSC and optical microscope results broadly tally with these findings (Table IV). The remainder of the work will concentrate on copolyesters IV-VII.

\section{Optical microscopy and phase identification of polyesters IV-VII}

Optical microscopy is a powerful tool in phase identification of liquid crystals. With molecular liquid crystals, often optical textures can be sufficient in identifying various liquid crystal phases and their transition temperatures. In the case of polymeric
Table IV. Glass Transition $\left(T_{g}\right)$, the Crystal-Nematic $\left(T_{K-N}\right)$, and Nematic-Isotropic $\left(T_{N-I}\right)$ Temperatures of Polyesters III-VII

\begin{tabular}{lrrrl}
\hline Polymer & $T_{\mathrm{g}}$ & $T_{K-N}$ & $T_{N-I}$ & Comments \\
\hline I & - & $\sim 600$ & - & degrades \\
II & - & $\sim 600$ & - & degrades \\
III & 140 & 350 & 455 & ref. 8 \\
III & - & 335 & 450 & ref. 5 \\
III & 132 & 333 & 450 & this work \\
IV A & 131 & 275 & 340 & this work \\
IV B & 136 & 295 & 470 & this work \\
V & 151 & 307 & 440 & this work \\
VI & 136 & 280 & 451 & this work \\
VII & 126 & 299 & 477 & this work \\
VIII & - & - & - & infusible \\
IX & - & - & - & infusible \\
X & - & - & - & infusible \\
\hline
\end{tabular}

All temperatures are in centigrade and correspond to the onset $\left(T_{g}\right)$ or peak temperatures in the DSC thermograms in Figure 5. $T_{N-I}$ will be slightly lower than the clearing temperature observed by microscopy.

liquid crystals, it may be possible to observe a fluid phase that shows birefringence, but it is often difficult to obtain a recognizable texture. This is evident in some of the reported literature on thermotropic liquid crystal polymers, where the textures have a mottled appearance, lacking in clarity. ${ }^{11-17}$ In some of these works, it is the $\mathrm{x}$-ray experiments rather than the optical textures that provide the decisive evidence for phase identification. ${ }^{12,18}$ Yet, in other works, textures from which phases can be unambiguously identified are shown. ${ }^{19-22}$

As hot-stage $\mathrm{x}$-ray facilities were not available, optical microscopy has been used for providing the primary information on phase identification of the mesophases in polyesters IV-VII. For this, we ensured that good textures were obtained in all cases to allow unambiguous identification of the phases. The polymer was fluid at the temperature and time the photographs of the textures were recorded; this could be detected by motion of the liquid or the movement of extraneous impurity particles. The DSC thermograms can be regarded as secondary evidence and will be presented after the microscopy results.

\section{Polyester IV}

Figures 1-3 shows the textures obtained from polyester IV A. Figure 1 is of a type referred to as a marbled texture by Demus and Richter ${ }^{23}$ and is often found in nematics. This was obtained immediately after isotropization and cooling to $315^{\circ} \mathrm{C}$. Marbled 


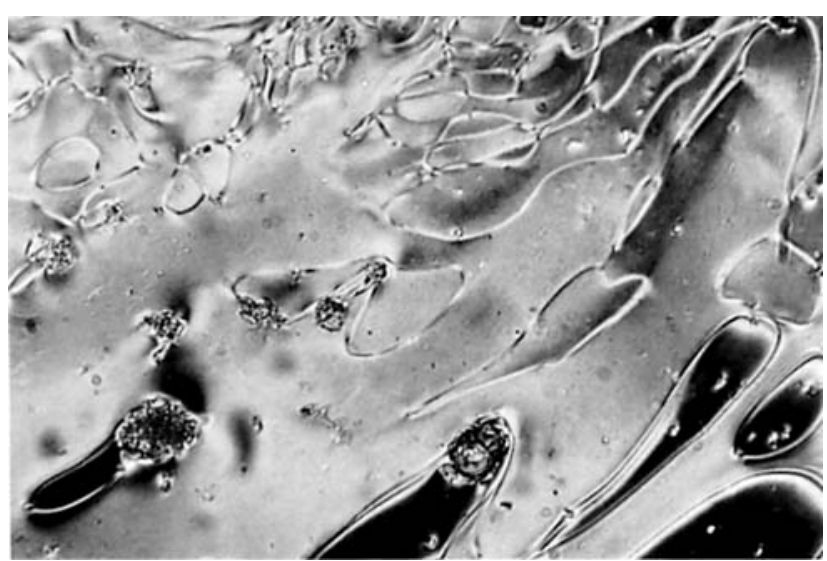

Figure 1. Marbled texture of the nematic phase of polyester IV A showing areas of near homeotropic alignment (dark zones). Crossed polars, $315^{\circ} \mathrm{C}$.

textures often appear with glass surfaces that have not been specially treated. The boundaries that separate different areas generally consist of threads, inversion walls of various types, or twist disclination lines. ${ }^{23}$ There was considerable movement of the fluid and the closed loops in Figure 1 could be seen floating in the liquid. These were unstable and disappeared after some time. ${ }^{23}$ The dark inclusions in the texture of Figure 1 are areas of near homeotropic alignment, while the colored zones arise from regions with a nearly defect-free planar alignment. When the hot stage was tapped gently, bright flashes in these dark areas were observed, due to the temporary collapse of the director from perpendicular to parallel alignment with respect to the glass slides. This is also a characteristic of the nematic phase and it often helps with phase identification. ${ }^{23}$

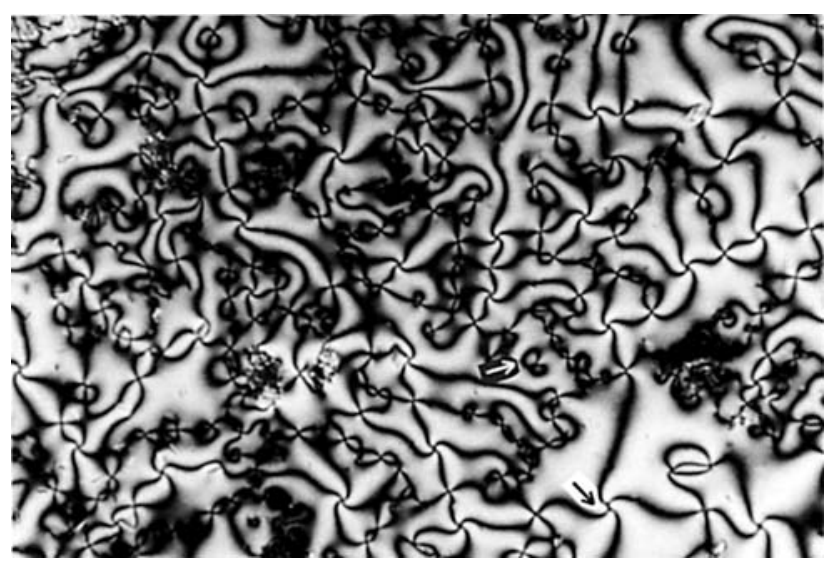

Figure 2. Schlieren texture of the nematic phase of polyester IV A. White arrow shows point singularities with $\mathrm{s}=\frac{1}{2}$; black arrow shows singularity with $\mathrm{s}=1$. Crossed polars, $330^{\circ} \mathrm{C}$.

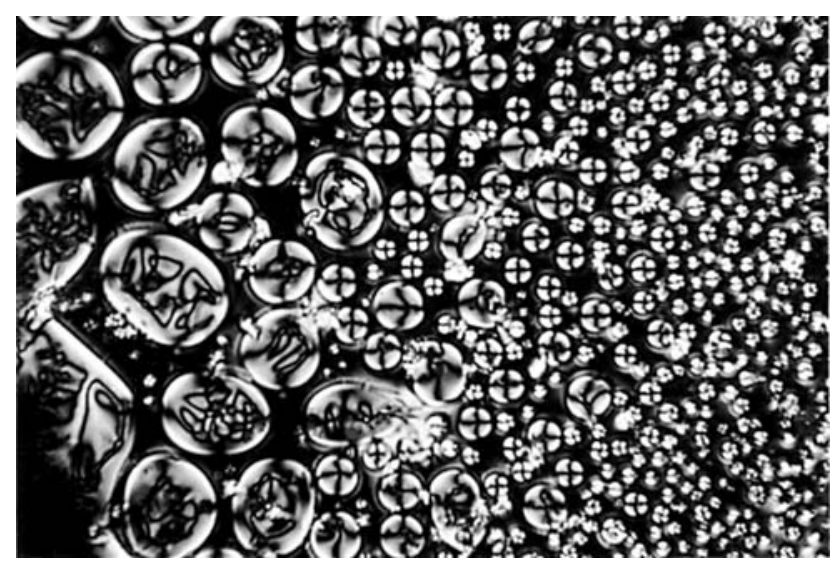

Figure 3. Droplet texture of the nematic phase of polyester IV A, emerging from the isotropic phase. Crossed polars, $365^{\circ} \mathrm{C}$.

Figure 2 shows a second type of texture obtained with polyester IV A. This was recorded at $330^{\circ} \mathrm{C}$ after cooling from the isotropic state. It can be seen that point singularities with strengths $\mathrm{s}= \pm \frac{1}{2}$ and $\mathrm{s}$ $= \pm 1$ are both present and this is sufficient to identify the phase unambiguously as nematic. ${ }^{24}$ Note that schlieren textures are also characteristic of smectic C phases; however, in these, singularities with $\mathbf{s}= \pm \frac{1}{2}$ do not occur and only those with $\mathrm{s}=$ \pm 1 are found. ${ }^{24}$

Figure 3 displays another texture characteristic of nematics that was observed with polyester IV A at $365^{\circ} \mathrm{C}$, while cooling from the clearing temperature. This is the droplet texture that is observed normally when the nematic phase emerges from the isotropic. ${ }^{21,23}$ The droplets typically showed extinction crosses, spiral structures, bipolar arrangements and other topologies. Theoretical studies confirm the stability of radial or bipolar orientations. ${ }^{25}$ The droplets grew and coalesced into other textures. With polyester IV A, such a droplet texture was observed, not only when the sample was cooled from the isotropic state as in Figure 3, but also at the transition from the nematic to the isotropic.

The higher molecular weight polyester IV B also showed schlieren and marbled textures characteristic of the nematic phase. However, the homeotropic and the droplet textures found with the lower molecular weight version IV A were not observed with IV B. Homeotropic orientation of the homopolyester of phenyl hydroquinone and phenyl terephthalic acid has been reported by Garay et al. ${ }^{10}$ but the cause is not well understood. It has been suggested however that homeotropic orientation is more likely to occur with lower molecular weight polymers due to the increased number of chain ends. ${ }^{26,27}$ The observation 


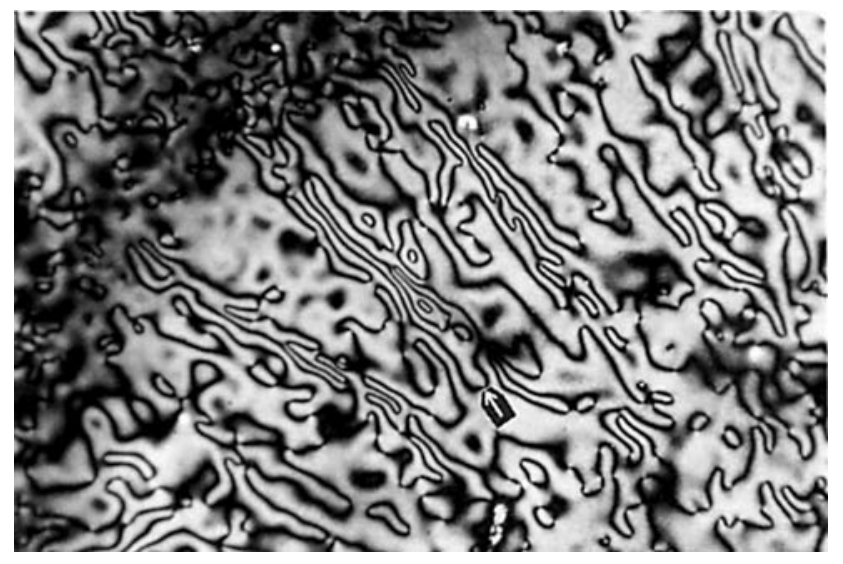

Figure 4. Texture of polyester $V$ showing surface inversion walls, ending at point singularities with $s= \pm \frac{1}{2}$. Crossed polars, $412^{\circ} \mathrm{C}$.

of the homeotropic orientation with polyester IV A, but not IV B, tends to support this view.

Two other observations are worth reporting. Firstly, cooling the mesophase even at $5^{\circ} \mathrm{C} / \mathrm{min}$ or $10^{\circ} \mathrm{C} / \mathrm{min}$ did not lead to any crystallization textures. When a molecular liquid crystal such as pazoxyanisole is cooled from the nematic phase, crystallization eventually occurs, and this can be easily detected optically because the nematic texture is "overwritten." Instead, with both polyesters IV A and IV B, the schlieren or the threaded texture was simply frozen into a glassy state, presumably because the crystallization rate was very slow.

Secondly, it was observed that prolonged holding (over $60 \mathrm{~min}$ ) at $330^{\circ} \mathrm{C}$, or above the clearing temperature for a shorter period, led to degradation. The degradation product, however, was not the charred material that was found if the sample was held above the clearing temperature for long periods. Instead, it appeared to be crystalline, taking the form of birefringent rods and diamond-shaped crystals (not shown). We have observed this phenomenon with other high-temperature polyesters and it is most probably due to the formation of the diacid resulting from the hydrolysis of the ester. This can give a misleading impression of a liquid crystal texture growing from the isotropic phase.

\section{Polyesters V-VII}

Polyester $\mathrm{V}$ was liquid crystalline in the temperature regime $307-440^{\circ} \mathrm{C}$ ( Table IV). Again, the best textures were obtained by rapidly heating above the clearing point $\left(440^{\circ} \mathrm{C}\right)$, and cooling quickly. Figure 4 shows a texture from polyester $\mathrm{V}$ that was predominantly populated with surface inversion walls ${ }^{23}$ (also called inversion walls of the first kind by
Nehring and Saupe ${ }^{24}$ ), emerging and ending at $\pm \frac{1}{2}$ point singularities. There is a $\pi / 2$ turn of the molecular director at the center of the wall. ${ }^{24}$ From the $\pm \frac{1}{2}$ point singularities (arrowed in Fig. 4), it is possible to conclude unambiguously that the mesophase was nematic.

Polyester VI formed a mesophase in the interval $280-451^{\circ} \mathrm{C}$ (Table IV). Again, a schlieren texture similar to Figure 2 with \pm 1 and $\pm \frac{1}{2}$ point singularities was formed (not shown) and once more it was possible to conclude unambiguously that the mesophase was nematic. Polyester VII showed both marbled and schlieren textures that again indicated a nematic mesophase between about $300^{\circ} \mathrm{C}$ and $475^{\circ} \mathrm{C}$. None of the polyesters V-VII showed the droplet or the homeotropic textures.

\section{DSC Thermograms}

Figure 5 shows the heating behavior of the copolyesters IV-VII recorded at the fastest heating and cooling rates attainable $\left(40^{\circ} / \mathrm{min}\right)$ to minimize degradation. It can be seen that all the polymers show two endotherms, one at about $300^{\circ} \mathrm{C}$ due to the crystal-nematic $(\mathrm{K}-\mathrm{N})$ transition, and another near or above $400^{\circ} \mathrm{C}$ that is due to the nematicisotropization ( $\mathrm{N}-\mathrm{I})$ transition. These transitions corelate with the melting behavior observed with the polarizing microscope. The glass transition temperatures, and the $\mathrm{K}-\mathrm{N}$ and $\mathrm{N}-\mathrm{I}$ transition temperatures obtained from DSC are listed in Table IV.

Double-melting endotherms, however, are not a sufficient criterion for the detection of LC phases in polymers, for such behavior can also be observed with non-liquid crystalline polymers. ${ }^{28,29}$ There are

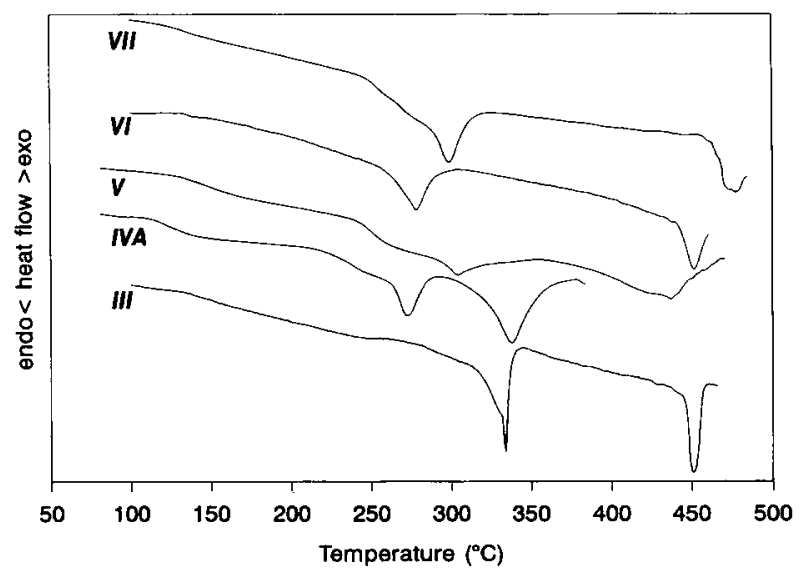

Figure 5. DSC heating curves of polyesters III-VII. All samples show two endothermic peaks. The lower-temperature peak is due to the $\mathrm{K}-\mathrm{N}$ transition and the second peak is the $\mathrm{N}-\mathrm{I}$ transition. In some cases, degradation follows immediately after the N-I transition. 


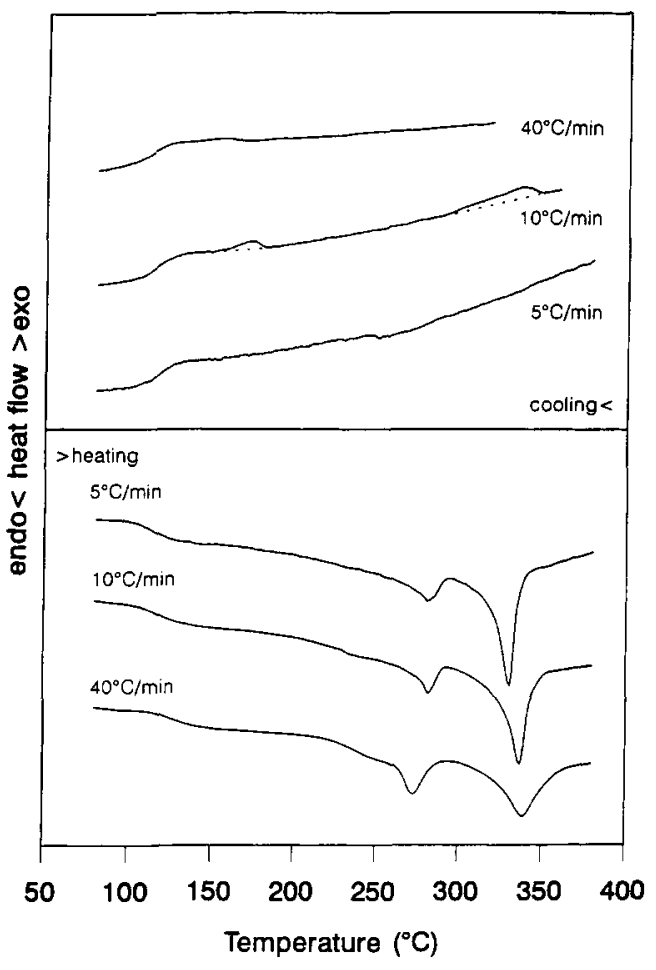

Figure 6. DSC thermogram of polyester IV A, showing the effect of heating and cooling rate. The bottom curves are the heating cycle and the associated cooling curves are shown on top.

examples in the literature citing the observation of more than one melting peak with polymers such as polyethylene ${ }^{30}$ or polybutylene terephthalate during the heating scan. ${ }^{31}$ Multiple endotherms can occur during the heating of such flexible-chain polymers due to the phenomenon of annealing (crystal thickening) during the heating scan. The annealing effect is heating-rate dependent; thus, whereas single crystal-melting peaks are observed at very low and very high heating rates, multiple peaks are found at intermediate heating rates. ${ }^{28}$

In the present case, crystal thickening is unlikely to occur in polymers with rigid chains, and hence the two principal endotherms in Figure 5 are likely to be due to two separate phase transitions. Note that with other types of LCPs that contain mesogens coupled with flexible spacers we have found the thermograms of the heating scan were difficult to analyze because peaks due to annealing effects also occur and are superimposed on mesophase transitions.

On cooling (after isotropization) at $40^{\circ} \mathrm{C} / \mathrm{min}$, no exothermic peaks were observed in the DSC with all the polyesters. There are two possible interpretations for this: there had been so much degradation at the isotropization temperature that no isotropic- to-nematic exotherm was observed, or else quenching at $40^{\circ} \mathrm{C} / \mathrm{min}$ led to an isotropic glass.

Heating and cooling rate effects will be illustrated by the behavior of one polymer, polyester IV A. Figure 6 (bottom) shows the DSC behavior of polyester IV $A$ at heating rates of $40^{\circ} \mathrm{C} / \mathrm{min}, 10^{\circ} \mathrm{C} / \mathrm{min}$, and $5^{\circ} \mathrm{C} / \mathrm{min}$. It can be seen that the two peaks that we have attributed to the $\mathrm{K}-\mathrm{N}$ and the $\mathrm{N}-\mathrm{I}$ transitions are present at low, intermediate, and high heating rates, suggesting that the two peaks are not merely due to annealing effects but are due to two consecutive phase transitions. Note, however, that the $\mathrm{K}$ $\mathrm{N}$ peak shifts to slightly higher temperatures at slower heating rates.

On the cooling scan (after isotropization), Figure 6 (top) shows that at a cooling rate of $40^{\circ} \mathrm{C} / \mathrm{min}$, there were no phase transitions, implying that the material had quenched to an isotropic glass. However, Figure 6 (top) shows that at a cooling rate of $10^{\circ} \mathrm{C} / \mathrm{min}$, two small exothermic transitions were observed, indicating that the phase transitions observed on the heating scan were indeed reversible. From the cooling scan at $10^{\circ} \mathrm{C} / \mathrm{min}$ in Figure 6 (top), the $\mathrm{I}-\mathrm{N}$ transition is at $333^{\circ} \mathrm{C}$ (peak temperature) and this corelates with the microscope observations. Note that the I-N phase change does not show supercooling. The $\mathrm{N}-\mathrm{K}$ transition in the cooling scan at $10^{\circ} \mathrm{C} / \mathrm{min}$ occurred at $174^{\circ} \mathrm{C}$; by comparing with the $\mathrm{K}-\mathrm{N}$ transition, it can be seen that there is considerable supercooling during crystallization. A similar lack of supercooling in the transition from isotropic to mesophase and a strong supercooling effect during crystallization has been reported for other LCPs. ${ }^{16}$ Finally, in the sample heated and cooled at $5^{\circ} \mathrm{C} / \mathrm{min}$, no exothermic peaks were observed on cooling (Fig. 6, top), though two peaks had been found on heating (Fig. 6, bottom). This was because the material was not thermally stable at elevated temperatures and very slow heating led to degradation. Thus, at this rate, a second heating cycle (after the first heat-cool cycle) revealed no endothermic transitions as the material had undergone degradation.

\section{Molecular Structure and Phase Transition Temperatures}

All the glass and phase transition temperatures of the polyesters that are collected together in Table IV can now be related to the molecular structures of the constituents.

With polyester III, comparisons can be made with the data in the literature. It can be seen that our transition temperatures for this polymer are com- 
parable to those of Krigbaum and Tanaka ${ }^{8}\left(333^{\circ} \mathrm{C}\right.$ vs. $\left.350^{\circ} \mathrm{C}\right)$ and Payet $^{5}\left(333^{\circ} \mathrm{C}\right.$ vs. $\left.335^{\circ} \mathrm{C}\right)$. Differences may arise because in some works the peak temperatures in the DSC are cited whereas the clearing temperatures from microscopy are quoted in others. The latter would generally be somewhat higher because it is taken as the temperature at which birefringence vanishes completely. There may be other factors, such as molecular weight and heating rate, that lead to differences when comparing the transition temperatures in the literature for the same polymer.

The concept of frustrated chain packing mentioned by Griffin and $\mathrm{Cox}^{2}$ is effective in lowering the transition temperatures of aromatic polyesters with all-para linkages. Thus, if polyester III is compared with I or II (Table I), it can be seen that the unsymmetrical structure with a single phenyl group on the hydroquinone suppresses the crystallinity and melting point by virtue of two effects: firstly, by the steric hindrance of having a large phenyl substituent, and secondly, by the random head-to-head or headto-tail addition of this monomer along the polymer chain.

In contrast to mono-substitution, symmetrical disubstitution of the hydroquinone with even large substituents such as phenyl groups does not appear to reduce crystallinity in the homopolymers (compare polymer III with VIII-X, Table 1) and hence these polymers did not melt. In the case of the 2,5 di-substituted polymers (VIII and IX in Table I), there is no differentiation between head-to-head or head-to-tail additions. However, in the case of the 2,6 di-substituted polymer (X, Table I), such a differentiation is possible, yet it was not sufficient to destroy the geometrical regularity and supress crystallization.

The copolyesters IV-VII can be considered to be similar to the homopolyester III, except that a third monomer (the di-substituted hydroquinones in IVVI or hydroquinone in polymer VII) has been added in small amounts to create a random copolymer.

Whereas the homopolyester III of hydroquinone and terephthalic acid had a crystal-nematic transition at $333^{\circ} \mathrm{C}$, all the copolymers IV-VII had the crystal-nematic transition at a lower temperature (in the vicinity of $300^{\circ} \mathrm{C}$, Table IV). The homopolyester III had a clearing temperature of $450^{\circ} \mathrm{C}$. Interestingly, all the copolyesters (except the lower molecular weight IV A) also had isotropization temperatures in the vicinity of $450^{\circ} \mathrm{C}$, but in two cases the N-I transition was actually higher. Thus, the addition of the di-substituted hydroquinone comonomer depresses the crystal-nematic transition while not having the same effect on the nematic- isotropic transition, when compared with the homopolymer III.

Comparing the data for the low and high molecular weight polyesters IV A and IV B, it is interesting to notice that the crystal-nematic transition is almost invariant at about $275-295^{\circ} \mathrm{C}$ but the clearing temperatures were drastically different (about $380^{\circ} \mathrm{C}$ for IV A vs. $480^{\circ} \mathrm{C}$ for IV B ). Thus, molecular weight seems to have a significant impact on the clearing temperature but not on the crystal-nematic transition.

Compared with the homopolymer, random introduction of a third monomer to form a copolymer introduces further disruption of chain regularity (apart from that caused by the bulk of the substituent, and head-to-head or head-to-tail addition where applicable). This also reduces crystallinity, even if the third monomer is the unsubstituted hydroquinone (polymer VII, Table I) or the symmetrically di-substituted hydroquinones as in polymers IV-VI. The latter is effective in disrupting chain packing when incorporated in small amounts due to the greater bulkiness of two substituents and the randomness of the inclusions. Though the copolymers IV-VI have lower crystal-nematic transition temperatures than the homopolymer III when the comonomer is added randomly in small amounts, it may be anticipated that in larger quantities, the incorporation of the di-substituted hydroquinone may create blockiness that would again increase the crystallinity and the melting point.

Generally, it appears that the polyesters made from the frustrated chain packing method will always tend to be nematic. This seems to be the case here. Polyesters made from a similar concept reported in other studies also appear to be exclusively nematics. ${ }^{10,12-14}$ In contrast, smectic mesophases are often formed from main-chain aromatic polyesters containing flexible spacers between mesogens. ${ }^{16,32}$ This is because in the former, the mesophase is stabilized by disrupting the regularity of the chain by unsymmetrical substitution, whereas in the latter, it is created by reducing the rigidity of the backbone while keeping a chain structure that is symmetrical. Nematics would be preferred to smectic polyesters for applications such as injection molding and fiber spinning owing to the lower viscosity of the former.

\section{CONCLUSIONS}

The copolyesters of terephthalic acid, phenylhydroquinone, and di-substituted hydroquinones melted and formed a liquid crystal phase. Optical microscopy was used as the primary tool to evaluate the 
nature of the mesophases. All the copolyesters showed a nematic liquid crystal phase; there was no multiple mesophase behavior. The classical features of the nematic phase, such as the marbled and the schlieren textures, as well as surface inversion walls, were observed with these polyesters. The droplet texture of the nematic phase and homeotropic alignment was observed only with a low molecular weight polymer.

The crystal-nematic transitions were generally in the vicinity of $300^{\circ} \mathrm{C}$. A clearing temperature, which is not always observed with aromatic polyesters, was found with all the copolyesters. The clearing temperatures were generally above $400^{\circ} \mathrm{C}$. The thermal stability in air at temperatures near or above the clearing point over a prolonged period was relatively poor, but as the nematic-to-isotropic range was over a $100^{\circ} \mathrm{C}$ in all the cases, a useful working range is still available with these copolyesters. To observe a crystal-nematic-isotropic sequence and the reverse isotropic-nematic-crystal (or glass) transition, fast heating and cooling rates had to be used.

The DSC results also confirmed the presence of first-order phase transitions that were in general accord with those observed by microscopy. Endothermic transitions corresponding to crystal-nematic and nematic-isotropic phase changes were recorded on the first heating run, with a fast heating rate. The observation of the reverse exothermic isotropic-nematic transition was not always possible due to degradation after isotropization.

\section{REFERENCES}

1. S. G. Cottis, J. Economy, and B. E. Nowak, U.S. patent 3637595 (1972) to Carborundum.

2. B. P. Griffin and M. K. Cox, Br. Polym. J., 12, 147 (1980).

3. M. Ballauf and G. F. Schmidt, Mol. Cryst. Liq. Cryst., 147, 163 (1987).

4. W. J. Jackson, Jr., Contemporary Topics Polym. Sci., 5, 177 (1984).

5. C. R. Payet (to Dupont) U.S. patent 4, 159, 365 (1979).

6. W. Jackson, Jr., Br. Polym. J., 12, 154 (1980).

7. R. R. Luise, U.S. patent 4183895 (1980).

8. W. R. Krigbaum and T. Tanaka, Macromolecules, 21, 743 (1988).
9. J. F. Harris, Jr., U.S. patent 4, 294, 955 (1981).

10. R. O. Garay, P. K. Bhowmik, and R. W. Lenz, J. Polym. Sci., Part A, Polym. Chem., 31, 1001 (1993).

11. C. K. S. Pillai, D. S. Sherrington, and A. Sneddon, Polym. Commun., 33, 3968 (1992).

12. P. K. Bhowmik, E. D. T. Atkins, and R. W. Lenz, Macromolecules, 26, 447 (1993).

13. R. L. Johnson and S. Z. D. Cheng, Macromolecules, 26, 94 (1993).

14. D. Dainelli and L. L. Chapoy, Macromolecules, 26, 385 (1993).

15. P. J. Stenhouse, E. M. Valles, S. W. Kantor, and W. J. MacKnight, Macromolecules, 22, 1467 (1989).

16. P. Meurisse, C. Noel, L. Monnerie, and B. Fayolle, Br. Polym. J., 13, 55 (1981).

17. S. W. Kantor and Ta-Cheung Sung, Macromolecules, 26, 3758 (1993).

18. P. K. Bhowmik, E. D. T. Atkins, and R. W. Lenz, Macromolecules, 26, 440 (1993).

19. A. M. Ghanem, L. C. Dickinson, R. G. Porter, and A. E. Zachariades, J. Polym. Sci., Part B, Polym. Phys., 28, 1891 (1990).

20. P. Le Barney, J. C. Dubois, C. Friedrich, and C. Noel, Polym. Bull., 15, 341 (1986).

21. G. Galli, M. Laus, A. S. Angeloni, P. Ferruti, and E. Chiellini, Eur. Polym. J., 21, 727 (1985).

22. C. Noel, C. Friedrich, F. Laupretre, J. Billard, L. Bosio and C. Strazielle, Polymer, 25, 263 (1984).

23. D. Demus and L. Richter, Textures of Liquid Crystals, Verlag Chemie (Weinham), New York, 1978.

24. J. Nehring and Saupe, J. Chem. Soc. Faraday Trans., II 68, 1, (1972).

25. a) E. Dubois-Violette, O. Parodi, J. Phys. Colloq., 30, C4-57 (1969). b) R. B. Meyer, Phys. Rev. Lett., 22, 918 (1969).

26. R. B. Meyer, in Polymer Liquid Crystals, A. Cifferri, W. R. Krigbaum and R. B. Meyer (eds.), Academic Press, New York, 1982.

27. R. W. Lenz, A. Furukawa, P. Bhowmik, R. O. Garay, and J. Majnusz, Polymer, 32, 1703 (1991).

28. J. Runt and I. R. Harrison, in Methods of Experimental Physics, vol. 16, part B, R. A. Fava (ed.), Academic Press, New York, 1980, p. 287.

29. Z. Bashir, J. Mater. Sci. Lett., 12, 598 (1993).

30. Z. Bashir, J. A. Odell, and A. Keller, J. Mater. Sci., 21, 3993 (1986).

31. M. E. Nichols and R. E. Robertson, J. Polym. Sci., Phys. Ed., 30, 755 (1992).

32. W. R. Krigbaum and J. Watanabe, Polymer, 24, 1299 (1983).

Received February 4, 1994

Revised May 2, 1994

Accepted May 10, 1994 